\title{
La centrale idroelettrica del cotonificio Widemann (San Germano Chisone, Torino): prospettive di conoscenza, conservazione e valorizzazione
}

DOSSIER PATRIMONIO DELL'ELETTRICITÀ: CITTÀ E PAESAGGI ELETTRICI

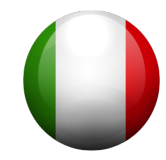

\section{Riccardo Rudiero}

PhD candidate. Politecnico di Torino, Dipartimento Architettura e Design. Torino [Piemonte] Italia $<$ riccardo.rudiero@polito.it>.

\section{Riassunto}

Le valli alpine del Pellice, Chisone e Germanasca, ai cui piedi sorge la città di Pinerolo (TO), furono tra le prime aree industrializzate dello stato sabaudo, vocazione che si riscontra ancora in un'ampia rete di testimonianze materiali: dai complessi produttivi alle opere sociali per la classe operaia; dalle infrastrutture di sfruttamento delle acque al sistema di elettrificazione. Riguardo quest'ultimo, sono ancora numerose le strutture atte alla generazione di energia elettrica, talune dismesse, altre in funzione, altre ancora in corso di trasformazione. Il presente contributo si soffermerà sul caso della centrale idroelettrica del Cotonificio Widemann di San Germano Chisone (TO), dove l'analisi dell'esistente e una lettura diacronica dei documenti d'archivio hanno permesso di ricostruirne la storia e hanno fornito la base per alcune suggestioni legate alla sua conservazione e valorizzazione.

\section{Parole chiave}

Impianto idroelettrico. Progetti. Archivio. Conservazione. Valorizzazione.

\section{The hydroelectric power plant of the Widemann cotton mill (San Germano Chisone, Turin): perspectives of knowledge, conservation and enhancement}

\begin{abstract}
Alpine valleys Pellice, Chisone and Germanasca, at whose feet lies the town of Pinerolo (TO), were among the first most industrialized areas of the Savoy state, vocation still spotted in the wide network of material evidences, such as production complexes, social facilities built for the working class, water channeling, electrification system. About the latter, there are still many active structures in the production of electricity. Some of them are dismantling, others are in operation, others are in the process of being transformed. This contribution will be focused on the case of the hydroelectric power plant of Widemann cotton mill in San Germano Chisone (TO), where the analysis of the existing structures and a diachronic reading of the archive documents has allowed to reconstruct its history and has provided the basis for some suggestions related to its conservation and enhancement.
\end{abstract}

\section{Keywords}

Hydroelectric power plant. Projects. Archive. Conservation. Enhancement. 


\section{Introduzione}

Le valli alpine del Chisone, Germanasca e Pellice, ai cui piedi sorge la città di Pinerolo (TO), comunemente conosciute come Valli Valdesi (Tourn, 2003), furono tra le prime aree maggiormente industrializzate dello stato sabaudo. Le attività tradizionali qui esercitate, legate all'agricoltura e all'allevamento, mutarono decisamente nei primi decenni del XIX secolo quando, grazie soprattutto all'abbondanza di acque e al rapporto con ambienti riformati europei (Bermond, 2014, p. 74), in diretto contatto con la realtà valdese locale, sorsero importanti iniziative imprenditoriali internazionali. Gli investitori stranieri, in particolare svizzeri e tedeschi, erano attratti dalle valli pinerolesi per la possibilità di accesso verso nuovi e promettenti mercati, con il vantaggio di un costo di manodopera inferiore rispetto ai loro paesi d'origine. L'avanzata tecnologia di cui disponevano - soprattutto in un settore in espansione come quello cotoniero - permetteva loro di mettere in piedi vantaggiose joint venture, dove i capitali maggiori erano investiti dagli imprenditori locali, a fronte della messa a disposizione dei macchinari e delle conoscenze sviluppate all'estero (Balcet, 2014, pp. 115-127).

Di queste realtà, che grandemente risentirono della crisi sorta a cominciare dagli anni Settanta dello scorso secolo, e che ha provocato dolorose dismissioni ancora nel recente passato ${ }^{1}$, si riscontra sul territorio un'ampia rete di testimonianze materiali: dai complessi produttivi alle opere sociali eseguite in favore delle popolazioni e della classe operaia; dalle infrastrutture di sfruttamento delle acque al sistema di elettrificazione.

A proposito di queste ultime, una recente indagine giornalistica ${ }^{2}$ ha messo in evidenza come nelle sole valli Chisone e Germanasca esistano 22 centrali idroelettriche - ancora funzionanti o che lo sono state nel recente passato - che derivano l'acqua dai torrenti per tramutarli in energia.

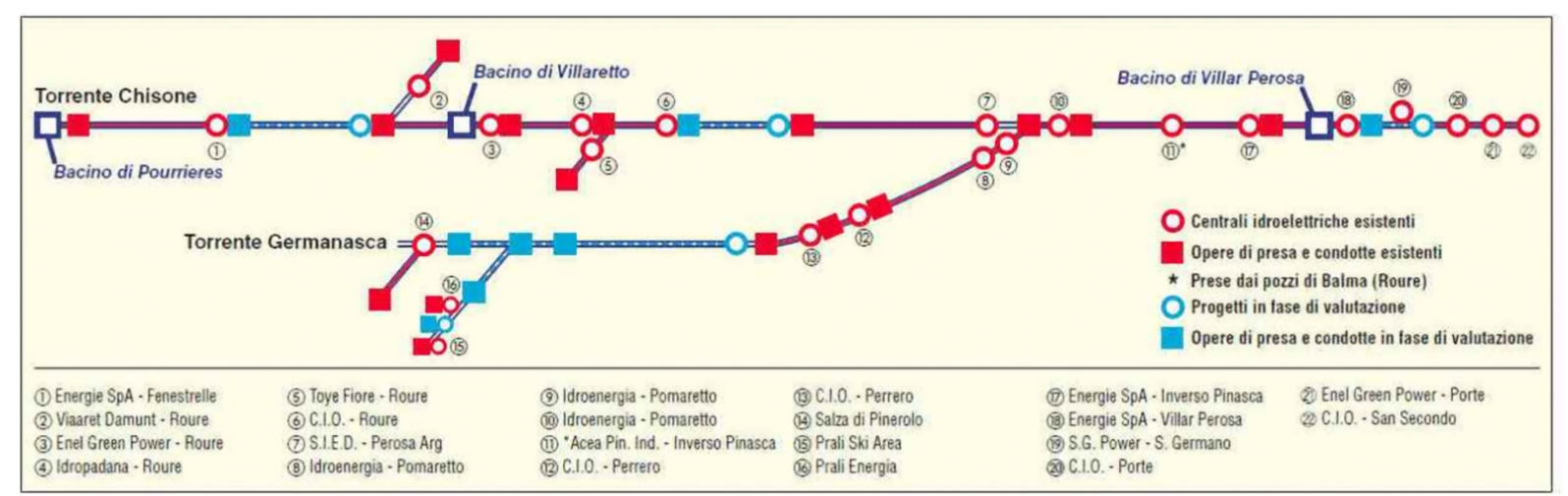

Figura 1. Indicazione schematica del posizionamento delle centrali idroelettriche nelle valli Chisone e Germanasca (da «L'Eco del Chisone» del 2 aprile 2014).

Se il boom di nuove installazioni si ebbe col Secondo Dopoguerra, i primi impianti idroelettrici sorsero sul finire del secolo XIX, quando i complessi produttivi se ne dotarono per favorire e incrementare le loro lavorazioni.

Tra le prime vi fu la centrale della Tuccia, in Val Troncea (collaterale della Chisone, dove nasce l'omonimo torrente), base della lavorazione della calcopirite estratta presso le miniere del Beth, tristemente celebri per le valanghe del 1904 che costarono la vita a 81 minatori (SIBILLA, 2014, pp. 42 e segg ). L'impianto fu frutto delle migliorie operate - a partire dal 1890 - dalla Compagnia Rami e Zolfi di Pinerolo; l'energia prodotta, circa $150 \mathrm{kwh}$, serviva per il trattamento del materiale e per

\footnotetext{
${ }^{1}$ Ad esempio, gli ultimi reparti della Gütermann, storico setificio perosino aperto nel 1870, chiusero nel 2001, mentre la Manifattura di Perosa (del Gruppo tessile cotoniero Manifattura di Legnano, ultimo detentore di quella che fu la Abegg, fondata come Jenny e Ganzoni sas nel 1883), cessò definitivamente la sua attività produttiva nel 2010.

${ }^{2}$ Assalto ai fiumi: affari d'oro con l'idroelettrcio, in «L'Eco del Chisone», mercoledì 2 aprile 2014, p. 15.
} 
l'impiego di perforatrici elettriche in galleria, oltre ad alimentare una teleferica lunga 4000 metri $^{3}$. Vi erano poi le centrali che sorgevano non per scopi industriali, ma per la sola fornitura di energia per l'illuminazione (e, in seguito, per altri usi domestici e/o produttivi). Il Piemonte fu pioniere in tal senso, grazie agli studi e alla produzione del piossaschese Alessandro Cruto, che permisero alla sua cittadina di dotarsi di illuminazione elettrica pubblica già nel 1883. Dieci anni dopo a Fenestrelle si costituì una società - prima nella valle Chisone - con l'intento di fornire la luce elettrica sia per l'illuminazione pubblica, sia per la privata (Bourlot, 2014, pp. 219-235).

\section{Tra ricerche d'archivio e materialità: l'impianto idroelettrico del cotonificio Widemann a San Germano Chisone (TO)}

Nei comparti industriali sorti nelle prossimità di centri abitati, la volontà di sfruttare l'energia idroelettrica coniugò i vantaggi industriali a quelli civili: migliorarono e aumentarono le produzioni ma, al contempo, si assistette a una progressiva elettrificazione pubblica e privata degli abitati cui le fabbriche afferivano.

Tra i primi (se non il primo) comparti che installò un impianto idroelettrico con finalità industriali, ma ne legò da subito le sorti anche alla pubblica illuminazione, fu quello del cotonificio di San Germano Chisone; il presente scritto vuol essere una breve sintesi di quanto ivi realizzato sul versante idroelettrico e di quanto, invece, rimase solo un progetto, basandosi sull'edito (Bottazzi \& Bounous, 1994) e sulla lettura di inediti documenti d'archivio4.

Il primitivo complesso, che sorgeva sull'area attualmente identificata ex Widemann (in virtù della famiglia che per ultima detenne per intero lo stabilimento), fu istallato sul finire degli anni Cinquanta del XIX secolo dal torinese Paolo Mazzonis, il quale scelse la val Chisone certamente per l'abbondanza delle acque e la possibilità di derivarle agevolmente. È stato ipotizzato come fattore determinante per la scelta specifica del sito il fatto che esistesse già un canale, costruito per il mulino comunale di San Germano, dal quale si sarebbe potuta convogliare l'acqua necessaria allo stabilimento (Bottazzi \& Bounous, 1994, p. 28). Certamente tale beale, che azionava sia il molino sia una manifattura di poco più a sud (l'opificio Davide Vinçon), giocò un ruolo per l'approvvigionamento idrico della nuova fabbrica, ma si provvide comunque alla costruzione di un canale specifico per il cotonificio. Questo è piuttosto chiaramente testimoniato dal Profilo longitudinale sul Canale in costruzione sino all'incontro del torrente Chisone, datato 1863 (ma sul quale è giustapposto un talloncino cartaceo con la dicitura «Copia conforme/Torino, 16 Luglio 1891»)5, attraverso il quale si comprende come Mazzonis decise subito di procurare una nuova derivazione verso lo stabilimento. La tesi troverebbe conferma in un ulteriore documento non datato, ma certamente anteriore al $1902^{6}$ (di cui si tratterà oltre), e nel quale si vede un canale denominato «del Cotonificio», diverso da quello del mulino, ma nel quale quest'ultimo si riversa.

La fabbrica avviò la produzione nel 1862, divenendo dagli anni Ottanta una delle più efficienti e grandi del Paese (Bottazzi \& Bounous, 1994, p. 34).

Mazzonis morì nel 1885, e la manifattura passò agli eredi, i quali lasciarono la direzione all'alsaziano Victor Widemann e a Carlo Simondetti - entrambi in carica da alcuni anni - che con oculatezza incrementarono la produzione. Nell'agosto 1892, tuttavia, la continua espansione della filatura venne bruscamente arrestata da un imponente incendio, e parve che i Mazzonis non volessero

\footnotetext{
3 http://www.pinographic.altervista.org/cesmap.html. Il complesso della Tuccia fu oggetto all'inizio degli anni Novanta di una campagna di scavi condotti dal CeSMAP (Centro Studi e Museo d'Arte Preistorica) di Pinerolo, insieme con la Soprintendenza Archeologica del Piemonte e l'Università di Torino. A proposito, si consiglia la visione del video presente all'indirizzo https://www.youtube.com/watch?v=P0UocPAuiN8

${ }^{4}$ Le ricerche sono state effettuate sull'Archivio Widemann (AW da qui in avanti) e sull'Archivio Storico Comunale (ASSGC), entrambi conservati presso il Comune di San Germano Chisone; la pubblicazione dei documenti in questa sede sarà, per ragioni di spazio, estremamente parziale e incompleta.

${ }^{5}$ AW, Serie 7, Sott. 1, F. 159 (Canale per forza motrice).

${ }^{6}$ Progetto di canale / da derivare dal Torrente Chisone / in territorio di S. Germano - Chisone / per aumento di forza motrice nel Cotonificio / WIDEMAN E SIMONDETTI. AW, Serie 7, Sott. 1, F. 159.
} 
investire fondi per la ricostruzione (Bottazzi \& Bounous, 1994, pp. 39-40). Nel luglio dell'anno successivo, quindi, vi subentrarono Widemann e Simondetti, confluiti in società, i quali acquistarono il complesso e si adoperarono affinché potesse tornare in esercizio. Dopo aver rinnovato la concessione governativa per la derivazione dal Chisone della forza motrice (Bottazzi \& Bounous, 1994, p. 42), essi fecero installare una prima centrale idroelettrica, alimentata anche dalle acque del canale del mulino (per l'affitto di quest'ultimo venne stipulato un contratto trentennale); come contropartita il Comune ottenne l'illuminazione elettrica - dal tramonto all'alba - per il centro del paese e lungo la strada del cotonificio, attraverso l'installazione di quattro lampade da sedici candele ad incandescenza, oltre la possibilità di una fornitura di energia ai privati che ne facessero richiesta7.

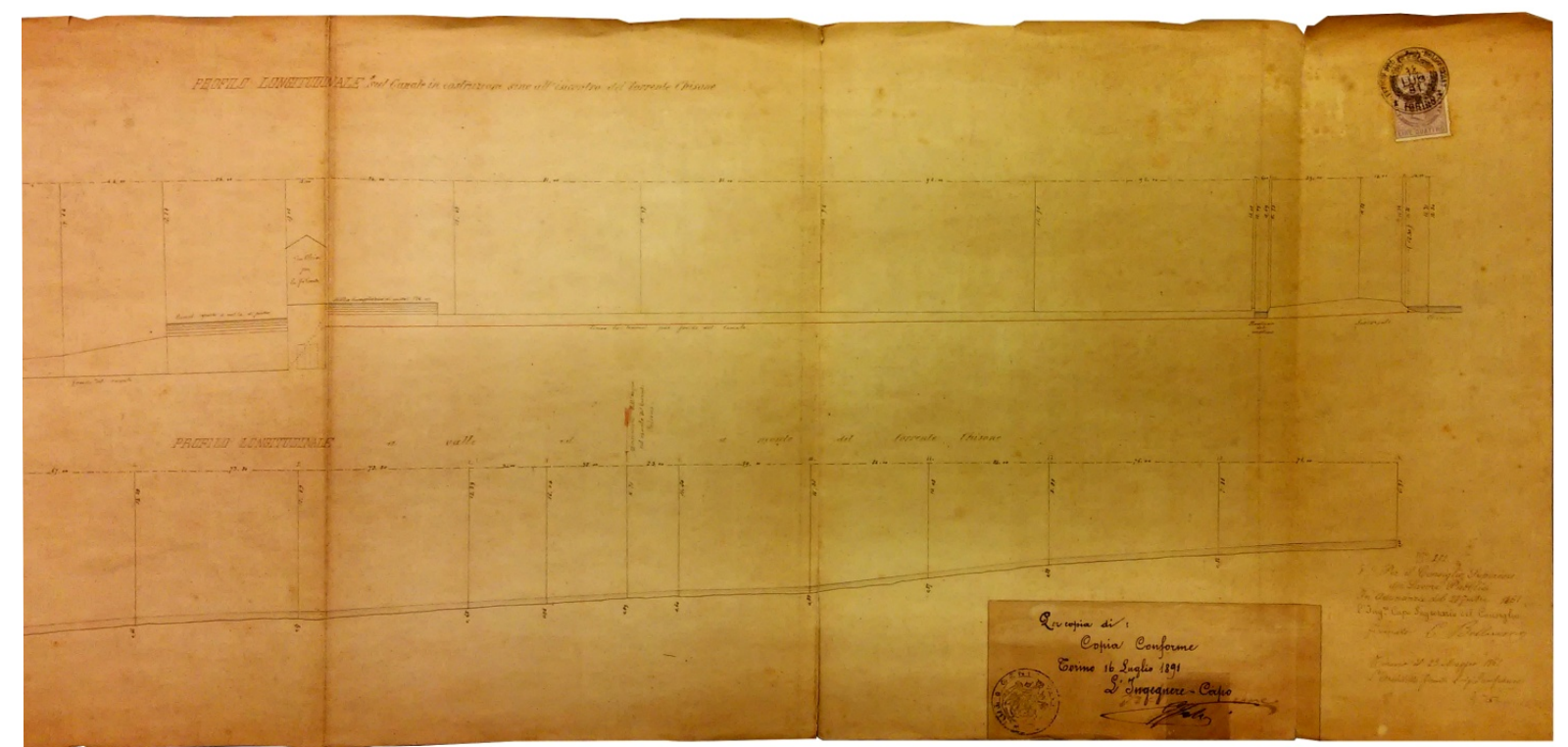

Figura 2. Estratto del profilo longitudinale del canale di adduzione al cotonificio Mazzonis, redatto nel 1863.

La riapertura della fabbrica venne celebrata con un pranzo il 25 febbraio del 1894 e, soprattutto, con l'inaugurazione dell'illuminazione pubblica a luce elettrica, che fu un vero e proprio evento mondano, come ebbero a scrivere i giornali dell'epoca (Bottazzi \& Bounous, 1994, pp. 7-8).

Nel 1902 Simondetti abbandonò la società, che entrò nella piena disponibilità di Widemann, il quale apportò progressivamente numerose migliorie - sia produttive che sociali - come il raccordo con la tramvia Pinerolo-Perosa (Bottazzi \& Bounous, 1994, p. 34; Avondo, Bruno, \& Seglie, 1998) ed il nuovo impianto di una turbina, per cui venne offerto un banchetto ai dipendenti del cotonificio il 4 novembre 1906 (Bottazzi \& Bounous, 1994, p. 46).

Per quasi un ventennio il Cotonificio agì quasi monopolisticamente sulle forniture elettriche comunali, almeno per ciò che concerne il capoluogo; tuttavia questo regime vantaggioso fu messo in discussione con il sorgere di altri importanti centri produttivi - che si dotarono di impianto idroelettrico - a monte e a valle dello stabilimento: nel 1906 nacque infatti la RIV di Villar Perosa e, un anno più tardi, la Società Talco \& Grafite in località Malanaggio, nel comune di Porte (Cerrato \& Ronchetta, 1996, pp. 191, 194)8.

Notizie generali sulle dotazioni tecniche del complesso industriale si hanno nel decennio post bellico (1915-1925), quando iniziarono le visite previste dalle disposizioni legislative della "Associazione Industriale d'Italia per prevenire gli infortuni sul lavoro": oltre alla generale arretratezza dei

\footnotetext{
${ }^{7}$ ASSGC, Serie 7, AS 46 (Affitto del mulino comunale).

${ }^{8}$ Risulta un dato di fatto che, a partire dall'inizio degli anni Dieci, vi fu una ripartizione della distribuzione dell'energia elettrica; ad esempio, il comune di Pramollo (confinante con San Germano, ma che vi fu accorpato dal 1928 al 1954) rimarrà tradizionalmente appannaggio della Società Talco \& Grafite, mentre quello di Inverso Porte (comune autonomo fino al 1928) di un susseguirsi di altre società, prima fra tutte la Società per le forze idrauliche dell'Alto Po (almeno dal 1912). ASSGC, AS 981.
} 
macchinari da lavoro, e alla mancanza dei più semplici dispositivi di sicurezza, si denota anche un pessimo giudizio sugli elementi dell'impianto elettrico, mantenuti in uno stato piuttosto precario (Bottazzi \& Bounous, 1994, p. 50).

La lettura dei documenti risalenti al periodo appena descritto chiariscono la visione d'insieme dei progetti e delle effettive realizzazioni riguardo lo sfruttamento dell'acqua per la produzione di energia elettrica del trentennio 1893-1924.

Per il primo decennio del XX secolo sono stati individuati due diversi progetti, entrambi intestati a Widemann e Simondetti, di cui uno datato 19019; il secondo, già citato in precedenza, non può essere fatto risalire oltre il 1902, data dell'abbandono della società da parte dell'italiano.

Entrambe le proposte progettuali caratterizzeranno una costante riscontrabile fino al Secondo Conflitto Mondiale, ossia la volontà di installare una centrale a monte ed una a valle del cotonificio, deviando il Chisone e prolungando il canale fin quasi alla località Malanaggio, a ridosso della (non ancora realizzata in quel periodo) Talco \& Grafite.

Il Progetto di prolungamento a valle del canale del Cotonificio di S. Germano Chisone, del 29 Gennaio 1901, propone di sfruttare la pendenza a sud dello stabilimento, sulla sponda destra del Chisone.

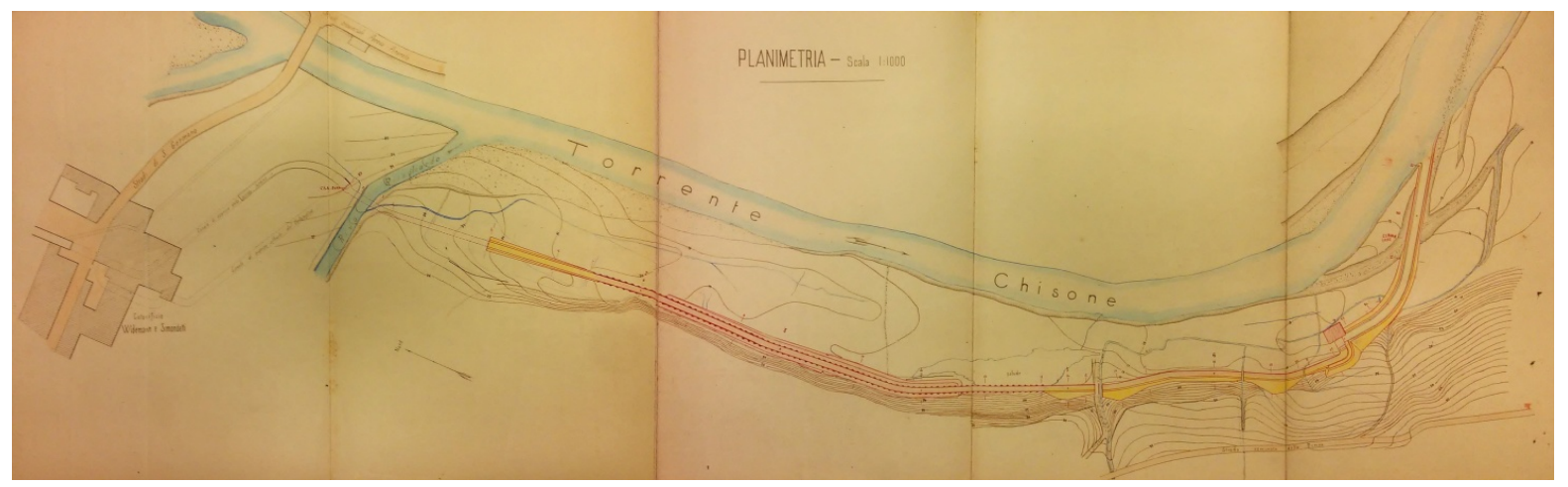

Figura 3. Planimetria generale (in scala 1:1000) del Progetto di prolungamento a valle del canale del Cotonificio di $S$. Germano Chisone del 1901.

Esso inaugura la stagione della famiglia Soldati come ingegneri progettisti per gli impianti idroelettrici del complesso sangermanese; in questo caso la firma è di Vincenzo, lo stesso che il 27 e 28 febbraio 1893 rilevò quanto sopravvissuto dall'incendio ${ }^{10}$.

Nel Progetto di canale da derivarsi dal Torrente Chisone in territorio di S. Germano Chisone per aumento di forza motrice nel Cotonificio Wideman e Simondetti11 si può invece vedere in planimetria il complesso delle derivazioni e la centrale elettrica, che avrebbe dovuto sorgere nei pressi del mulino. Nel disegno risulta già realizzato il canale del cotonificio (da me campito in azzurro), derivato quindi anteriormente al 1902. È da notare che il tracciato di quest'ultimo corrisponde per gran parte del percorso a quanto ancora oggi riscontrabile sul territorio; tuttavia manca il primo tratto, probabilmente distrutto da una piena e non più ripristinato. In effetti, potrebbe intendersi in tal senso quanto espresso nella Relazione allegata al Progetto Definitivo per Canale per forza motrice da derivare dal T. Chisone in territorio di S. Germano, del 26 Gennaio 192112 (l'ultimo redatto sotto Vittorio Widemann, che infatti morì nel 1924), opera dei fratelli Giacinto e Antonio Soldati. In esso si legge che «la nuova diga sul Chisone è fatta come opera instabile [...], con tipo

\footnotetext{
${ }^{9}$ Widemann \& Simondetti. Progetto di prolungamento a valle del canale del Cotonificio, di S. Germano Chisone. AW, Serie 7, Sott. 1, F. 159.

${ }^{10}$ Ditta Widemann \& Simondetti. Ricostruzione del Cotonificio di S. Germano - Chisone. AW, Serie 7, Sott. 2, F. 165 (Linee distribuzione energia elettrica).
}

11 AW, Serie 7, Sott. 1, F. 159. La mancanza della doppia finale nel cognome dell'alsaziano è un refuso presente sul documento.

12 AW, Serie 7, Sott. 1, F. 159. 
analogo alla diga del canale del Cotonificio Widemann, già esistente, ed asportata dalla piena 24 Settembre $1920 » 13$. Il tutto può essere ulteriormente suffragato dalla planimetria catastale intestata al figlio di Widemann, che dovrebbe rappresentare la situazione intorno agli anni Trenta del secolo scorso ${ }^{14}$, nella quale si evince planimetricamente la mancanza del tratto iniziale del canale.
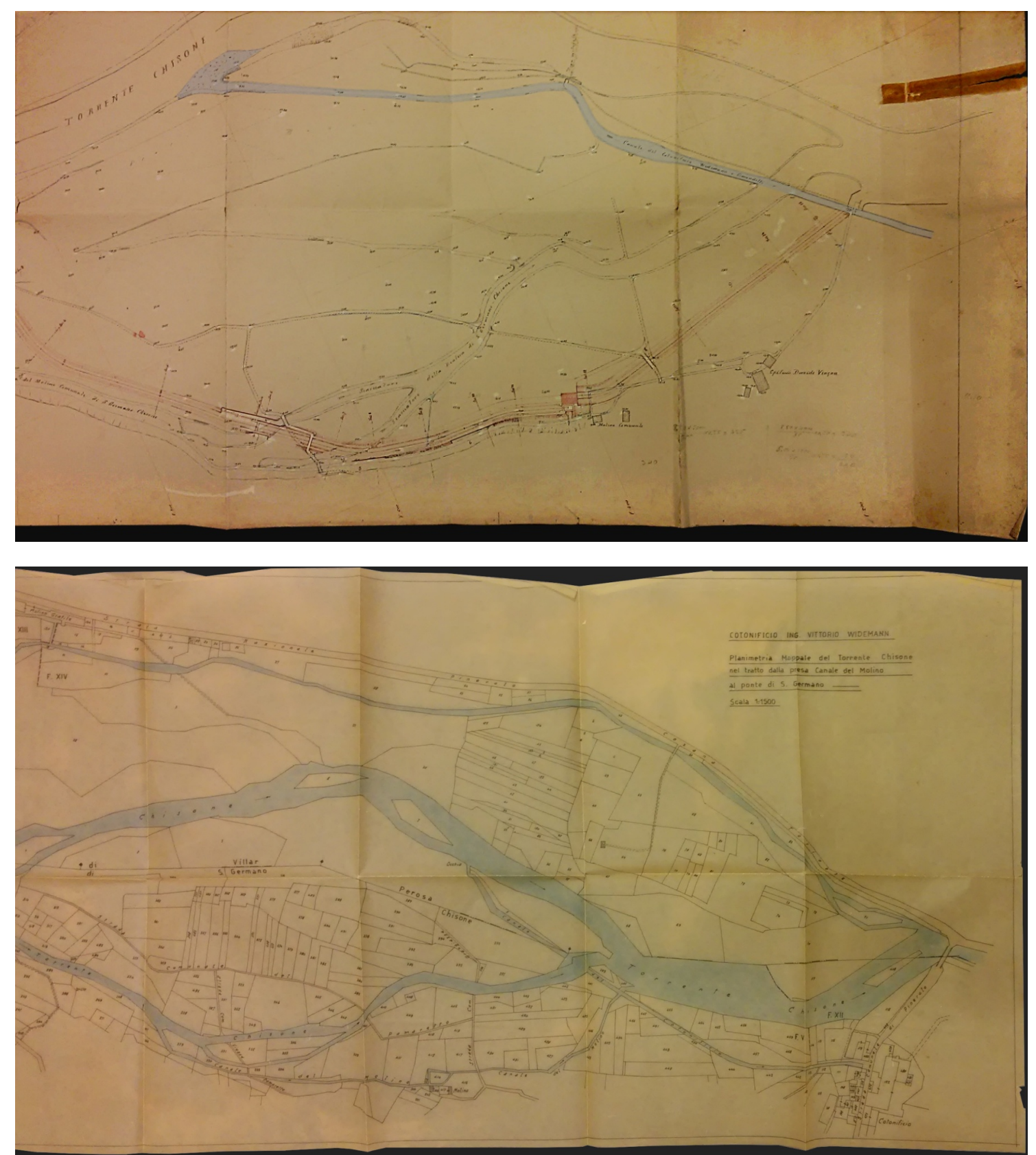

Figure 4 e 5. Confronto tra il progetto del canale a monte del cotonificio (1902 circa) e la Planimetria Mappale del Torrente Chisone (scala 1:5000, particolare), eseguita negli anni in cui la proprietà del complesso era di Vittorio Widemann Jr. Nel primo documento (estratto da planimetria in scala 1:1500) è stato da me evidenziato in azzurro il percorso del canale, che si discosta - per il tratto iniziale - dal più prossimo alla nostra epoca.

Con la morte di Widemann, la proprietà della ditta passò al figlio Vittorio Jr., il quale fu autore di numerosi cambiamenti. Già nel 1925 si eseguirono ristrutturazioni e nuove costruzioni; fu inoltre comprato il mulino comunale, compresi «tutti gli impianti meccanici di proprietà comunale, e l'impianto idraulico [...], nonché i diritti di derivazione d'acqua spettanti al comune di San Germano

13 AW, Serie 7, Sott. 1, F. 159, M. 159/1, Relazione e Computi.

14 AW, Serie 7, Sott. 1, F. 158 (Dighe e canali), M 158/1. 
Chisone [...]»15. L'acquisto impegnava Widemann a «[...] fornire gratuitamente nel comune la corrente elettrica necessaria per l'illuminazione dei locali comunali e delle strade» 16 , nonché di fornire ai privati che ne avrebbero fatta domanda «la corrente necessaria per l'illuminazione, purché non si trovino a distanza (non) superiore di mille metri in linea d'aria dalla Chiesa Valdese»17. Comprese nell'accordo, anche l'illuminazione delle borgate circostanti che, se più lontane del chilometro pattuito, avrebbero comunque pagato $10 \%$ in meno rispetto alle condizioni generali delle altre grandi società fornitrici di energia elettrica nel circondario18.

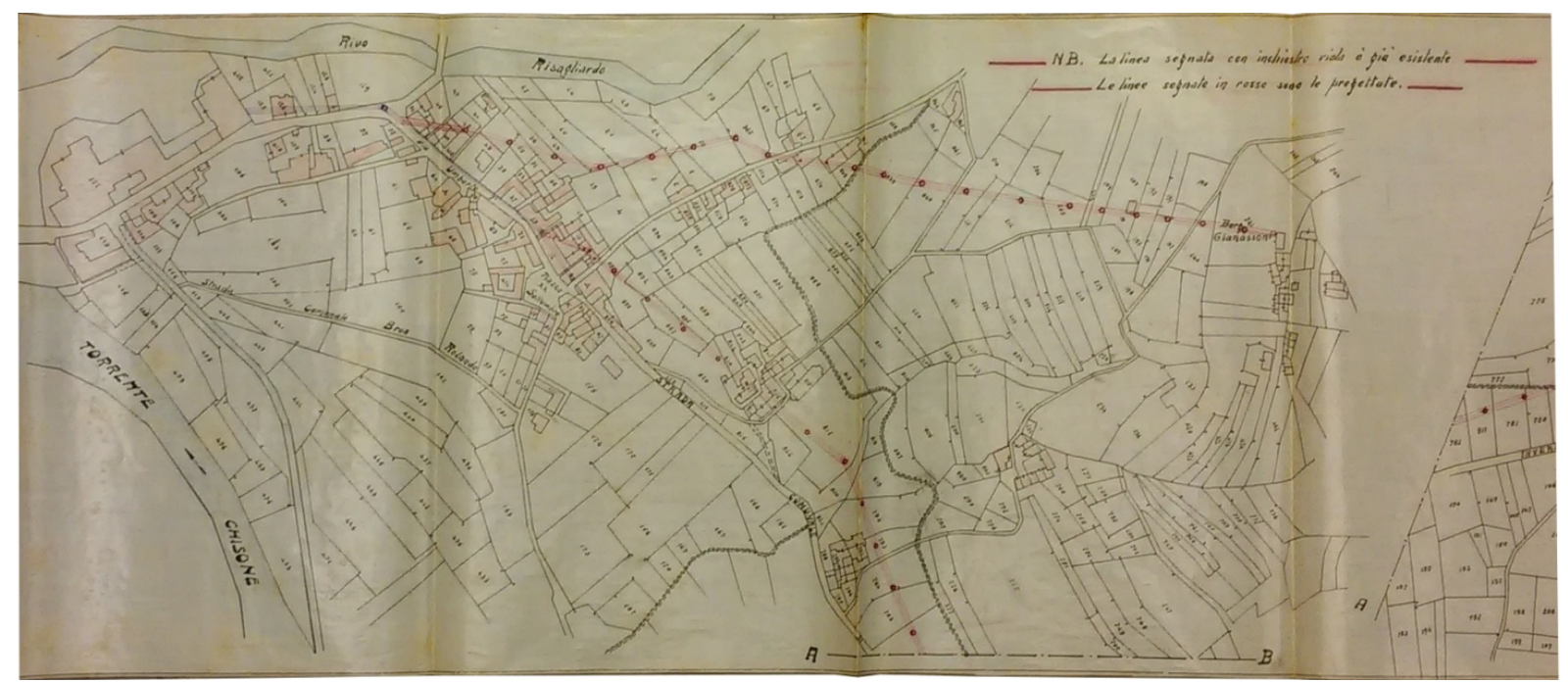

Figura 6. Estratto della Domanda per la/concessione impianto/linea di allacciamento/per fornitura luce (26/01/1928, scala 1:1500), in cui si vedono le linee esistenti e quelle in progetto negli anni della nuova espansione della fornitura nel Comune (AW, Serie 7, Sott. 1, F. 161).

Sempre in quegli anni, venne costruita una nuova centrale elettrica, «destinata a garantire la forza motrice e a permettere la sostituzione delle antiquate dinamo per l'illuminazione dello stabilimento con un moderno trasformatore» (Bottazzi \& Bounous, 1994, p. 52).

La crisi del 1929 si fece sentire anche in territorio italiano, e la ditta Widemann non fu esentata dalle sue ripercussioni; tuttavia, anche grazie al continuo uso di fibre naturali (per lo più cascami) in luogo delle più redditizie artificiali, essa si mantenne in buono stato di salute. Questa sua condizione venne certificata dalla pubblicazione edita dal regime fascista Viri ac res del 1935, che celebrava le eccellenze produttive nazionali, e a cui venne dedicata una pagina e mezza:

\begin{abstract}
I Widemann da industriali che considerano la loro industria [...] come un organismo economico di produzione autonoma e indipendente, non hanno voluto vassallaggi con altre industrie. Così per evitare una dipendenza onerosa dall'industria elettrica si sono creati due centrali elettriche, l'una di $500 \mathrm{Kw}$, l'altra di 200, quest'ultima di recentissima costruzione, entrambe azionate da energia idrica [...]. Il plus di trasformazione delle due centrali viene ceduto alle zone limitrofe, così gratuitamente il paese di S. Germano consuma l'energia elettrica Widemann, che è divenuta un sottoprodotto del cotone (Bottazzi \& Bounous, 1994, p. 64).
\end{abstract}

Per quanto abbia potuto verificare nell'archivio Widemann, non ho potuto riscontrare progetti che si riferiscano specificamente a questa nuova centrale, come neppure a quella precedentemente realizzata; tuttavia, in un rilievo generale del complesso eseguito nel $1970^{19}$, sono indicate la

15 ASSGC, Categoria X - Lavori pubblici, poste, telegrafo, telefono, radio; Classe 3 - Illuminazione e energia elettrica; F. 981 (Disposizioni, circolari e documenti relativi all'illuminazione pubblica). Repertorio 1070, minutaro 890. 7 febbraio 1926.

16 Ibid., art. 6.

17 Ibid., art. 7. La parentesi racchiudente il “non” è mia, poiché indica un chiaro refuso del contratto.

18 Ibid. Artt. 8 -9.

19 Cotonificio Ing. Vittorio Widemann/S. Germano Chisone/Planimetria generale scala 1:200. AW, Serie 7, Sott. 2, F. 165 (Linee distribuzione energia elettrica). 
centrale TIBB (evidenziata da me in blu sulla planimetria), da $160 \mathrm{KW}$ e $60,5 \mathrm{mq}$, e la centrale Siemens (in verde), da $276 \mathrm{KW}$ e $527 \mathrm{mq}$, entrambe con i relativi canali di adduzione e scarico, e che sono probabilmente quelle a cui si riferisce l'autore di Viri ac Res. Entrambe hanno sede nei corpi di fabbrica costruiti non oltre gli anni Trenta del XX secolo.

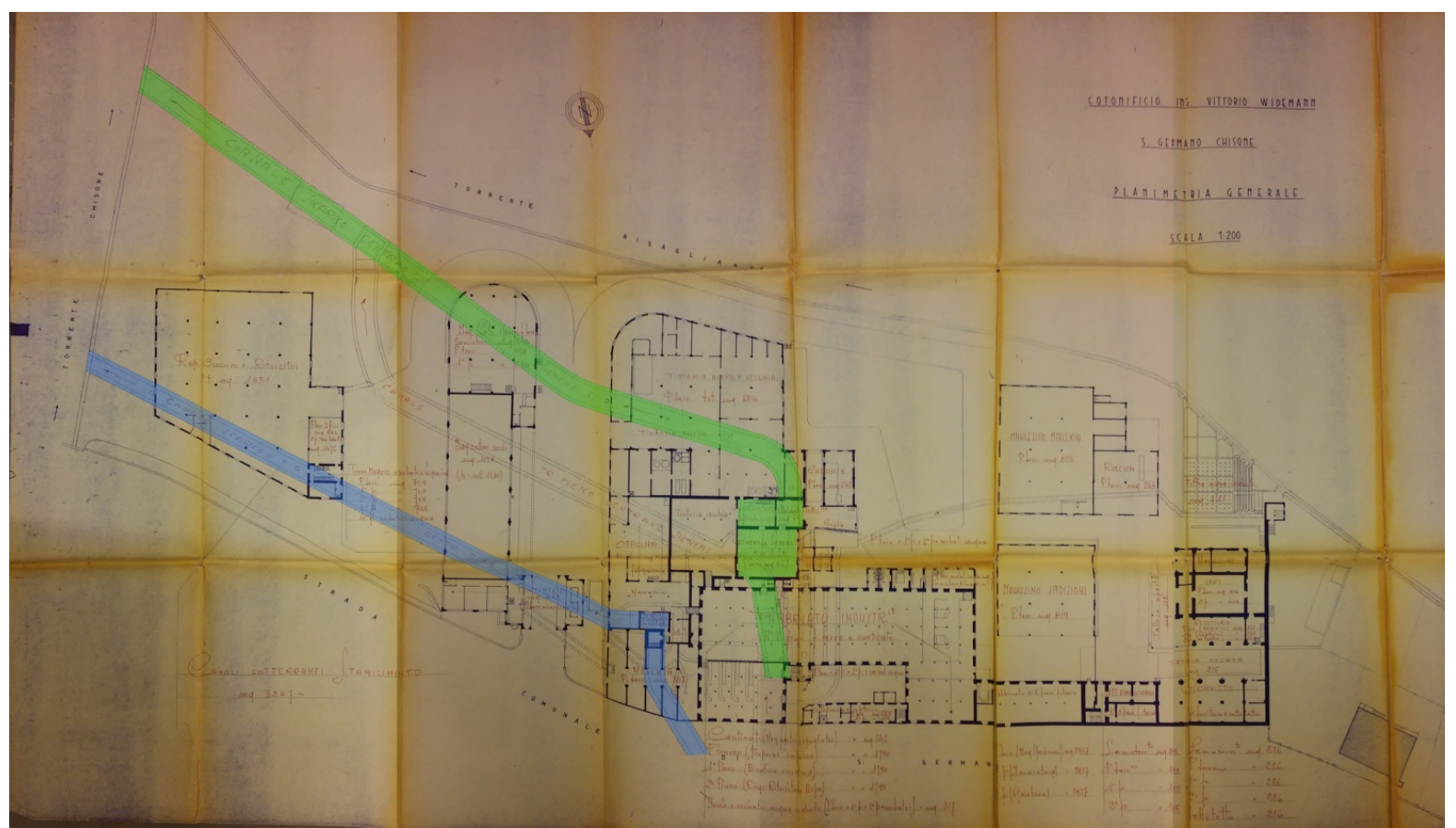

Figura 7. Le centrali e i relativi canali di adduzione e scarico presenti nel complesso, secondo il rilievo del 1970: in blu la TIBB e in verde la Siemens (i colori sono un'elaborazione sulla planimetria originaria in scala 1:200, di cui l'immagine presente è un estratto).

La volontà di Widemann di incrementare la produzione idroelettrica fu un punto fermo della sua gestione aziendale: a partire dai progetti del 1927, per giungere a quelli negli anni del Secondo Conflitto Mondiale, si evince l'intenzione di realizzare gli impianti sia a monte che a valle del cotonificio, sulla scorta delle proposte già operate in precedenza. La lettura di questi documenti fornisce interessanti informazioni sul progresso tecnico scientifico legato ai macchinari impiegati per la conversione della forza idraulica in elettrica e dà indicazioni sul mutare delle tecniche costruttive applicabili alle varie componenti dell'impianto (dal canale di adduzione alle centrali elettriche), compresi i materiali costruttivi.

Se, ad esempio, nel progetto Derivazione dal Torrente Chisone in prolungamento del Canale del Cotonificio per produzione di forza motrice, datato 15 gennaio 192720, il collegamento tra la camera di carico e il pozzo piezometrico avrebbe dovuto essere garantito tramite tubi di legno ( $\varnothing$ $1,70 \mathrm{~m}$ ), nella Variante del 6 settembre $1942^{21}$ la condotta forzata sarebbe stata metallica, e collegata alla centrale - per la prima volta collocata sulla sponda sinistra del Chisone - «a mezzo di un ponte di ferro, che è previsto del tipo sospeso, con catenaria formata da ferri piatti». Per ciò che concerne gli impianti a monte, invece, si segnalano quattro diversi progetti: del 23/02/1927 il primo ${ }^{22}$, del 18/02/193123 il secondo, del 17/12/193724 il terzo e infine del 18/02/194125,

20 AW, Serie 7, Sott. 1, F. 161 (Illuminazione pubblica: planimetrie). Variante al progetto esecutivo.

21 Variante all'impianto idroelettrico/derivato dal Chisone. Progetto di massima. AW, Serie 7, Sott. 1, F. 162, M. 162/3.

22 Canale per forza motrice da derivare dal T. Chisone in territorio di S. Germano - Progetto definitivo. AW, Serie 7, Sott. 1, F. 159, M 159/2.

23 Canale per forza motrice da derivare dal T. Chisone in territorio di S. Germano - Progetto esecutivo. AW, Serie 7, Sott. 1, F. 159, M 159/4.

${ }^{24}$ Impianto idroelettrico superiore - Progetto esecutivo. AW, Serie 7, Sott. 1, F. 162, M. 162/2.

25 Derivazione dal Torrente Chisone/in territorio di/San Germano - Progetto di massima. AW, Serie 7, Sott. 1, F. 159, M. $159 / 5$. 
tutti a firma di Antonio Soldati. I primi due e l'ultimo, sostanzialmente ricalcano le proposte del 1901 e del 1921; caso differente è il progetto del 1937, che prevede un andamento delle canalizzazioni alternativo e la centrale decisamente discosta rispetto al mulino.

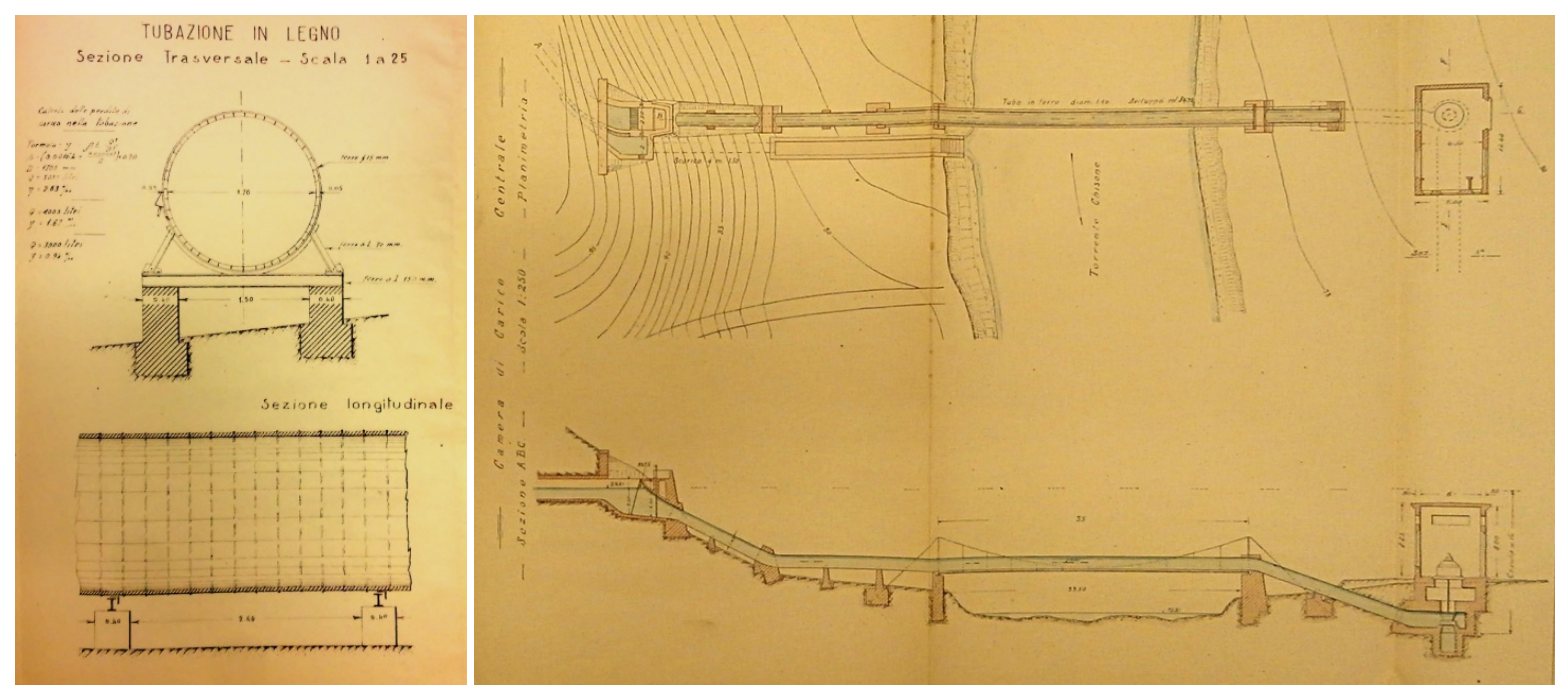

Figure 8 e 9. Prospetto e sezione delle tubazioni in legno previsti per la condotta forzata del progetto del 15 gennaio 1927 (scala 1:25); pianta e sezione del superamento del Chisone per mezzo di un ponte in ferro della condotta alimentante la centrale, secondo l'ipotesi del 1942 (scala 1:250).

Connesse a tutte queste opere, ma anche per la gestione di quanto già realizzato, in archivio sono presenti innumerevoli progetti per la tutela delle sponde del Chisone (come, ad esempio, il progetto di massima del 1927 Difese di sponda del Torrente Chisone ${ }^{26}$ ), e come testimonia, a partire dal Dopoguerra, il consorzio intercomunale sorto proprio per tale ragione.

Un altro fattore interessante riscontrabile negli archivi risulta essere la richiesta di preventivi e specifiche tecniche per i macchinari atti alla produzione elettrica vera e propria, con allegati progetti e fascicoli illustrativi; la più interpellata sembrerebbe essere la ditta "Costruzioni Meccaniche Riva" di Milano - tra le prime produttrici dell'epoca - consultata già dai primi anni ' $30^{27}$.
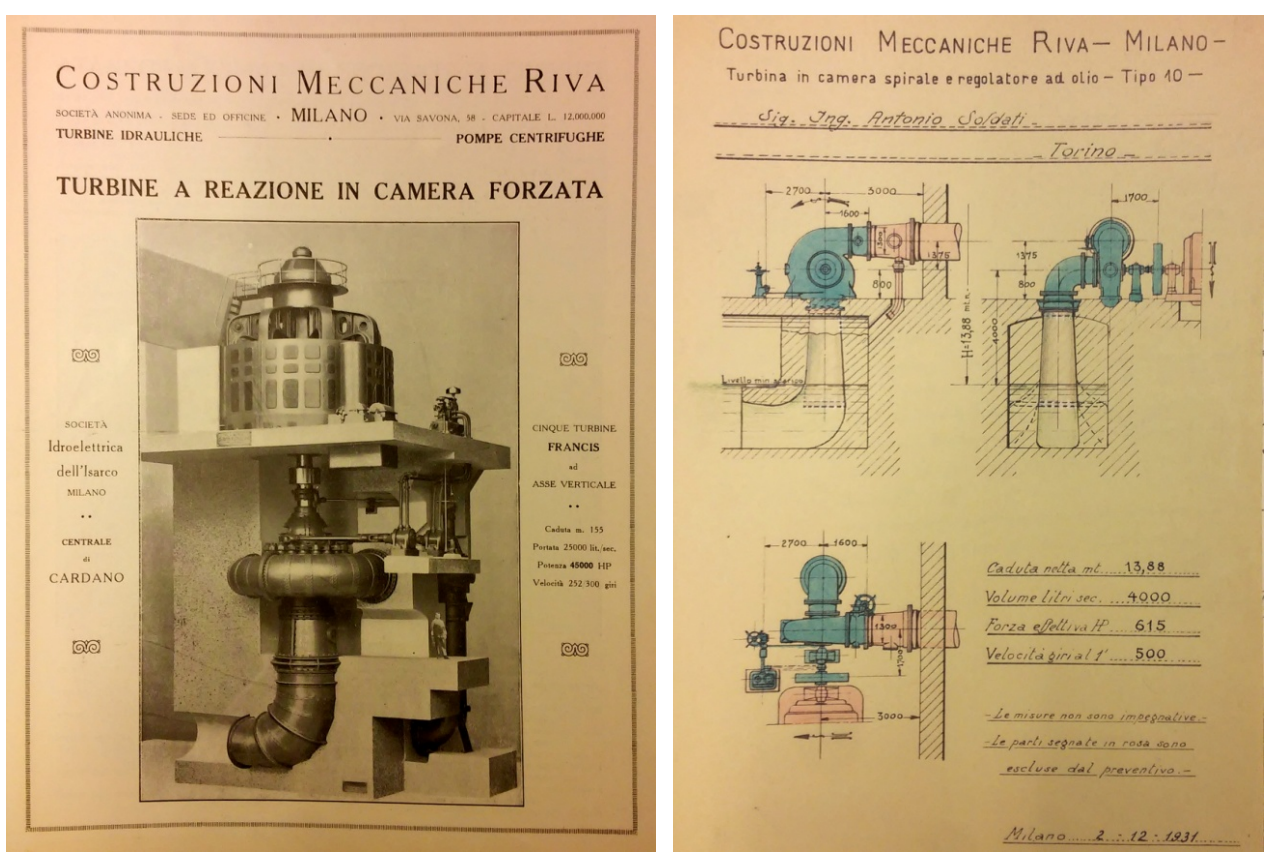

Figure 10 e 11.

Materiale

informativo e progetto per una Turbina in camera spirale e regolatore ad olio - Tipo 10 $(02 / 12 / 1931)$ delle Costruzioni Meccaniche Riva.

\footnotetext{
${ }^{26}$ AW, Serie 7, Sott. 1, F. 158.

27 AW, Serie 7, Sott. 1, F. 162.
} 
Durante la Seconda Guerra Mondiale, Widemann attivò una massiccia campagna di acquisto dei terreni lungo la sponda del Chisone, da Villar Perosa allo stabilimento (Bottazzi \& Bounous, 1994, p. 67); questo ebbe ripercussioni anche sui progetti - gli ultimi presenti in archivio - del 30/07/1943, ancora a firma del Soldati, e del 15/06/194728, opera dell'Ingegner Gaetano Rusconi. Infatti, in entrambe le proposte, la linea produttiva si sarebbe interamente attestata in sponda sinistra del Chisone, traendo l'acqua a Villar Perosa e reimmettendola a Inverso Porte.

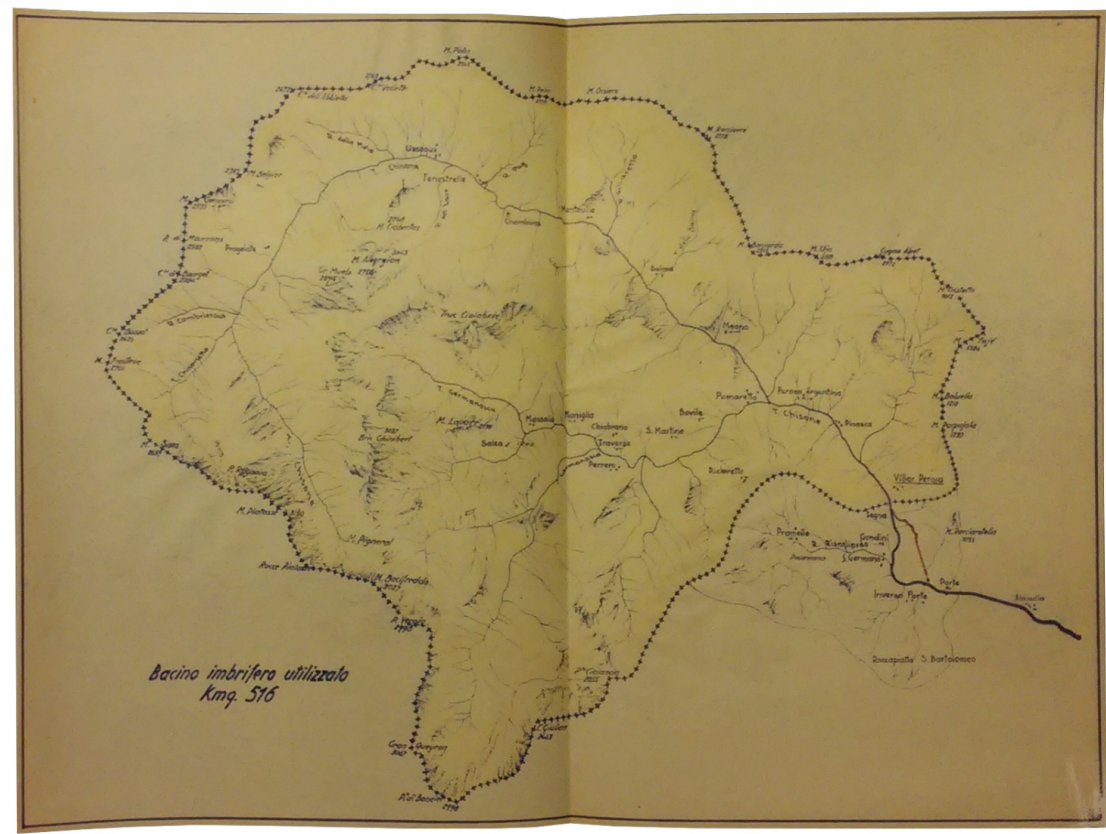

Figura 12. Bacino imbrifero potenziale per il progetto in riva sinistra del Chisone (il cui tracciato è segnalato in rosso nella planimetria originale) del 1947.

Vittorio Widemann morì nel 1966, e lo stabilimento fallì nel 1978; dopodiché venne lottizzato e destinato a diverse tipologie di produzione.

Degni di nota, per ciò che riguarda la componente della produzione elettrica, è il passaggio dell'impianto sangermanese all'ENEL nel 196729, con conseguenti rilievi e del sistema distributivo in tutta l'area, che dimostra la fitta ramificazione delle linee in più di settant'anni di attività.

\section{Dopo la dismissione: strategie di conservazione e valorizzazione del Cotonificio Widemann negli ultimi decenni.}

Dalla dismissione a oggi, è però importante sottolineare due tendenze - che hanno subito un'accelerazione dirompente soprattutto negli ultimi anni - inerenti la sostenibilità, dapprincipio culturale e legata alla conservazione della memoria, e poi energetica. Iniziando da quest'ultima, è almeno dalla fine dello scorso Millennio che esiste un programma complessivo di riuso di alcune centrali idroelettriche presenti sul Chisone e i suoi affluenti (Campra, 2000), e quella di San Germano rientra tra queste (progetto Idroval 4). La proposta - in corso di realizzazione - prevede la costruzione ex-novo di una centrale sull'impronta di quella esistente all'interno del complesso del cotonificio (la Siemes), e il recupero di parte del canale di adduzione, che però sarà realizzato in condotta forzata con tubi d'acciaio ${ }^{30}$. Saranno eseguite inoltre delle opere di compensazione, che

28 Derivazione per forza motrice/dal torrente Chisone/fra Villar Perosa e Porte - Progetto esecutivo. AW, Serie 7, Sott. 1, F. 159, M. 159/7.

${ }^{29}$ Cotonificio Widemann: trapasso impianto d'illuminazione pubblica all'Enel (Lettera del 19.4.1967). Serie 7, Sott. 1, F. 161 , M. 161/4.

30 Giunta Provinciale di Torino, Verbale n. 23, Adunanza 20 giugno 2014. Oggetto: Istruttoria interdisciplinare della fase di valutazione ai sensi dell'art. 12 della l.r. 40/1998 e s.m.i., relativa al progetto: "impianto idroelettrico denominato "Idroval 4" con derivazione d'acqua dal torrente Chisone. Protocollo: 444 - 20607/2014, p. 3 (http://www.cittametropolitana.torino.it/ cms/risorse/ambiente/dwd/via/provvedimenti-valutazione/val201-250/DGP_n.444-20607.pdf). 
prevedono tra l'altro la riqualificazione di una cospicua parte dell'area ex Widemann e la sistemazione della bealera dei Molini31.

Per quanto riguarda la prima, invece, è importante segnalare una serie di progressivi interventi volti dapprima alla conservazione delle testimonianze ancora presenti - dalle archivistiche alle paesaggistiche - e poi alla loro valorizzazione e pubblicizzazione. Infatti, la memoria del cotonificio è stata tutelata inizialmente attraverso l'intervento dalla Chiesa Valdese ${ }^{32}$, che si è fatta carico della conservazione dell'intero patrimonio archivistico, acquisito in seguito dal Comune. Oltre ciò, già dal 2002 l'amministrazione comunale ha dato luogo a una serie di itinerari storico-escursionistici legati alla fabbrica Mazzonis-Widemann; in particolare il "Sentiero Bianco" è un percorso che si snoda a partire dal parco della Villa Comunale (la quale ospita, tra l'altro, un'area in cui sono esposti alcuni reperti industriali locali) e lungo il sottostante canale del cotonificio, e che illustra in sintesi la storia dell'azienda dando particolare rilievo all'energia idrica. Lungo l'itinerario sono presenti vari pannelli, ciascuno dei quali documenta alcuni elementi caratteristici dell'ambiente naturale o le tracce dell'attività antropica. Il percorso raggiunge poi tutte le opere legate alla derivazione dell'acqua per il cotonificio (canali di derivazione, depuratore, paratoie, frantoio), per ritornare infine al complesso produttivo.
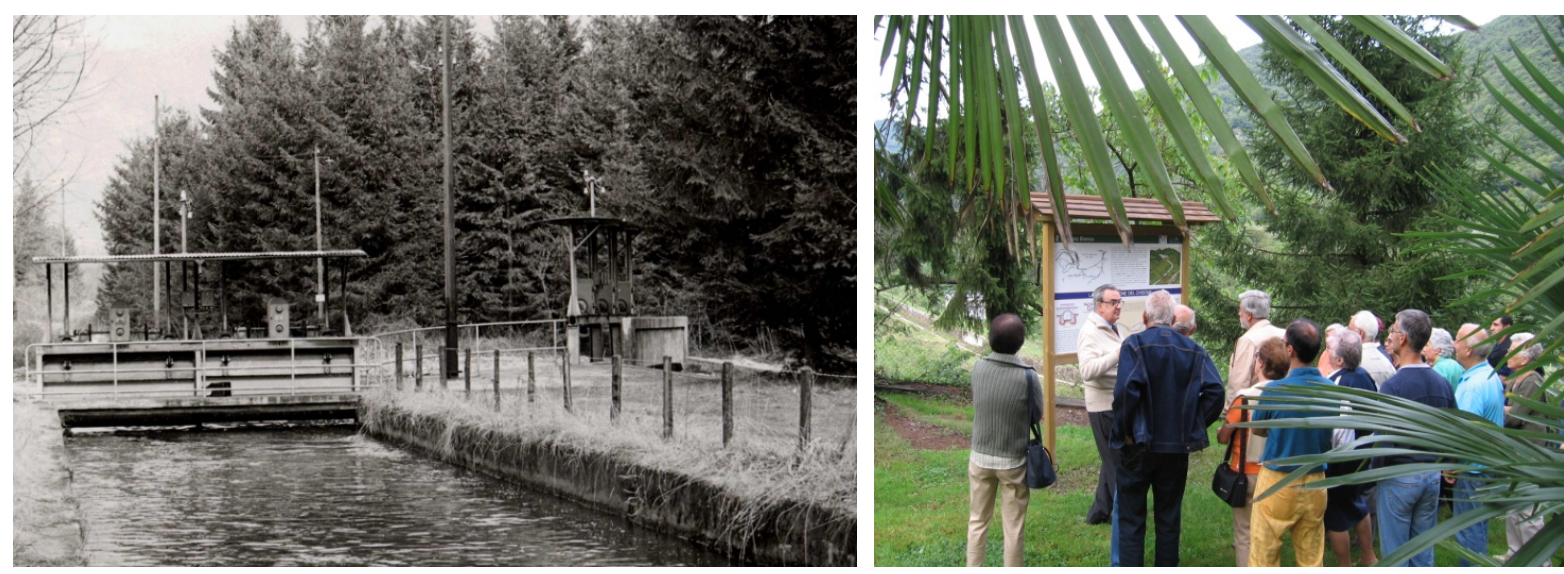

Figure 13 e 14. Paratoia del canale cotonificio, in una foto d'epoca, e gruppo di persone di fronte a un pannello illustrativo del "Sentiero Bianco", ove gli elementi funzionali vengono valorizzati en plein air (foto: http:// www.comune.sangermanochisone.to.it/index.php/gallerie-fotografiche/5-galleria-fotografica-2014/detail/217sentiero-bianco-medium.html?tmpl=component).

Certamente, i lavori intrapresi a partire dal 2015 implicheranno delle perdite rispetto a quanto ancora presente, se non altro perché il canale a cielo aperto verrà affiancato e/o sostituito da tubazioni, e il passaggio di queste ultime provocheranno in alcuni casi la rimozione dei sistemi di chiusa originari; tuttavia è importante che, sulla scorta di quanto già effettuato, e in una visione sostenibile a 360 gradi, si possa coniugare in maniera fruttuosa la tutela della memoria con la produzione di energia pulita, dove la seconda non mortifichi ma anzi accentui la portata socioculturale della prima.

\section{Le industrie delle Valli Valdesi come sistema: prospettive di conservazione e valorizzazione.}

A corollario delle valutazioni legate alla valorizzazione del patrimonio sangermanese, giacché non è possibile pensarlo scisso dal suo contesto più ampio, si vuole ora allargare il campo della riflessione sull'intero comparto industriale pinerolese e valligiano.

Sul fronte della conoscenza e della tutela, molti e autorevoli sono stati gli studi che hanno riguardato questa tipologia di beni culturali (Ronchetta \& Trisciuoglio, 2008; Massarente \& Ronchetta, 2004; Cerrato \& Ronchetta, 1996), che hanno portato non solo all'identificazione e alla catalogazione

\footnotetext{
31 Ibid., p. 7.

32 Per particolar merito della prof. Clara Bounous, già sindaco di San Germano, che inoltre ringrazio sentitamente per la disponibilità dimostratami durante questa ricerca.
} 
di tutti gli edifici produttivi, ma anche alla proposta di una serie di percorsi e itinerari che mettessero in evidenza, integrandoli tra loro, i valori storici, ambientali e fisici, con specifico riferimento alla loro cultura materiale. Attualmente, purtroppo, questi studi risultano essere un semplice repertorio giacché molti di questi complessi si stanno progressivamente abbattendo; da ciò si evince che il valore documentario di ciò che resta inizia a prevalere sull'aspetto analitico e pratico che inizialmente informava le suddette ricerche. Ciononostante, non sono poche le iniziative volte alla loro tutela e valorizzazione: a titolo esemplificativo, si ricordano il progetto "Ecomuseo delle miniere e della valle Germanasca" di Prali; l'associazione "Ecomuseo delle attività industriali di Perosa Argentina e valli Chisone e Germanasca" di Perosa Argentina; il percorso "Difiloinfilo", nel comune di Pomaretto; il museo "Ski Rochon" di San Germano Chisone, ove una piccola sezione è dedicata alle attività del cotonificio Widemann; il "Museo della Storia della meccanica e del Cuscinetto" di Villar Perosa. Tuttavia, nonostante l'abbondanza di progetti, anche dal comprovato valore culturale, si riscontra una disorganica gestione globale di questo patrimonio che ha radici comuni, e si invoca da più parti una messa a sistema per dare una svolta alla promozione turistico-culturale delle Valli.

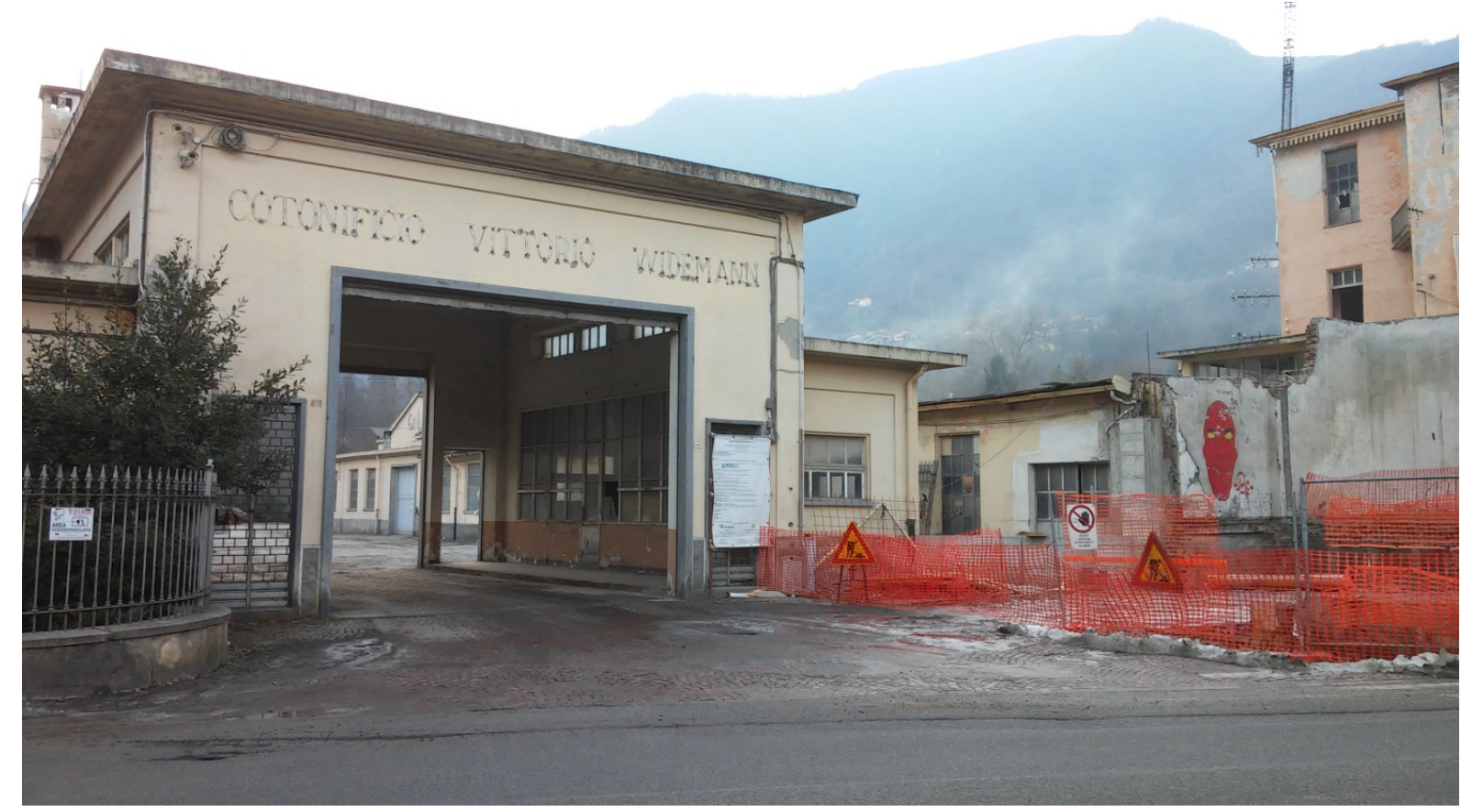

Figura 15. Cotonificio Widemann, dicembre 2016: lavori in corso per la creazione della nuova centrale idroelettrica (foto: Rudiero).

Per sopperire a ciò, a partire dal 2015 i Comuni della Bassa Val Chisone e Germanasca, in sinergia con associazioni e istituzioni culturali quali Italia Nostra e il CeSMAP di Pinerolo, stanno elaborando una proposta progettuale parimenti culturale e turistica per promuovere la conoscenza, conservazione e valorizzazione del patrimonio industriale valligiano, mettendo in rete le emergenze e sistematizzandole con le altre attrattive di natura diversa (storico-artistiche, naturalistiche, legate alla tradizione, ecc...). La proposta prevede di predisporre un progetto pluriennale che, partendo da un nucleo realizzabile in breve tempo e con un finanziamento non eccessivamente elevato, possa svilupparsi negli anni integrando azioni finalizzate a completare e migliorare le risorse presenti nella rete nei vari comuni che vi aderiscono.

Come primo atto, ciascun Comune o Ente preposto dovrà predisporre un aggiornamento dell'anagrafica dei beni e una valutazione della consistenza degli archivi; dopodiché si potrà passare alla messa a sistema delle iniziative già esistenti sul territorio. Grazie a questa fase propedeutica, si potranno ipotizzare azioni volte alla valorizzazione dei beni anche attraverso l'inserimento di nuove funzioni al loro interno. Preme sottolineare che la sistematizzazione dell'intero patrimonio, dove ciascun sito è considerato non isolatamente bensì come parte di un ambiente complesso, favorirà la differenziazione dell'offerta culturale e turistica delle Valli, evitando iniziative ridondanti che potrebbero causare la frammentazione del potenziale pubblico fruitore. 
Una volta espletata questa prima fase, si provvederà a un'azione coordinata a livello sovracomunale che dovrà prevedere:

- la creazione di uno o più archivi, sistematizzati tra loro, volti non solo alla conservazione della consistenza e della memoria dell'ambiente e delle fabbriche, ma anche e soprattutto dei lavoratori che ivi operarono;

- l'istituzione di un centro permanente di documentazione e di studi pluridisciplinari;

- l'approntamento di una comunicazione di tipo tradizionale (cartellonistica presente presso ogni sito che riconduce all'appartenenza al medesimo sistema culturale) e su altri media (internet, social network, ecc.) di matrice comune.

Si propongono a tal proposito:

- la realizzazione di un sito internet che convogli tutte le informazioni di ogni singolo ambiente, polo industriale e protoindustriale delle valli, facendo da piattaforma comune organicamente concepita;

- la produzione di materiale audiovisivo, spendibile non solo nei centri designati alla visita, ma anche online;

- l'ideazione di un percorso turistico attraverso l'uso delle nuove tecnologie che prevedano l'impiego di QRcode e Realtà Aumentata, anche ad uso dei disabili.

L'ipotesi di progetto prevede come attori principali gli abitanti delle aree prese in considerazione; si potrebbe quindi parlare di un sistema o museo diffuso che non mira alla valorizzazione esclusiva delle testimonianze materiali, ma anche degli ambienti e dei saperi locali. Le popolazioni residenti quindi rivestirebbero un ruolo di primo piano, essendo parte integrante del processo valorizzativo.

Grande attenzione s'intende altresì riservare all'attività rivolta agli studenti, dai primi gradi di istruzione fino alle secondarie superiori, affinché possano comprendere l'ambiente naturale e sociale, il processo industriale e il notevole peso che esso ebbe sulla formazione del paesaggio, anche attraverso visite sul campo e anche innovativi metodi didattici digitali e interattivi, da affiancare ai tradizionali e a esperienze conoscitive coinvolgenti di tipo laboratoriale pratico e ludico.

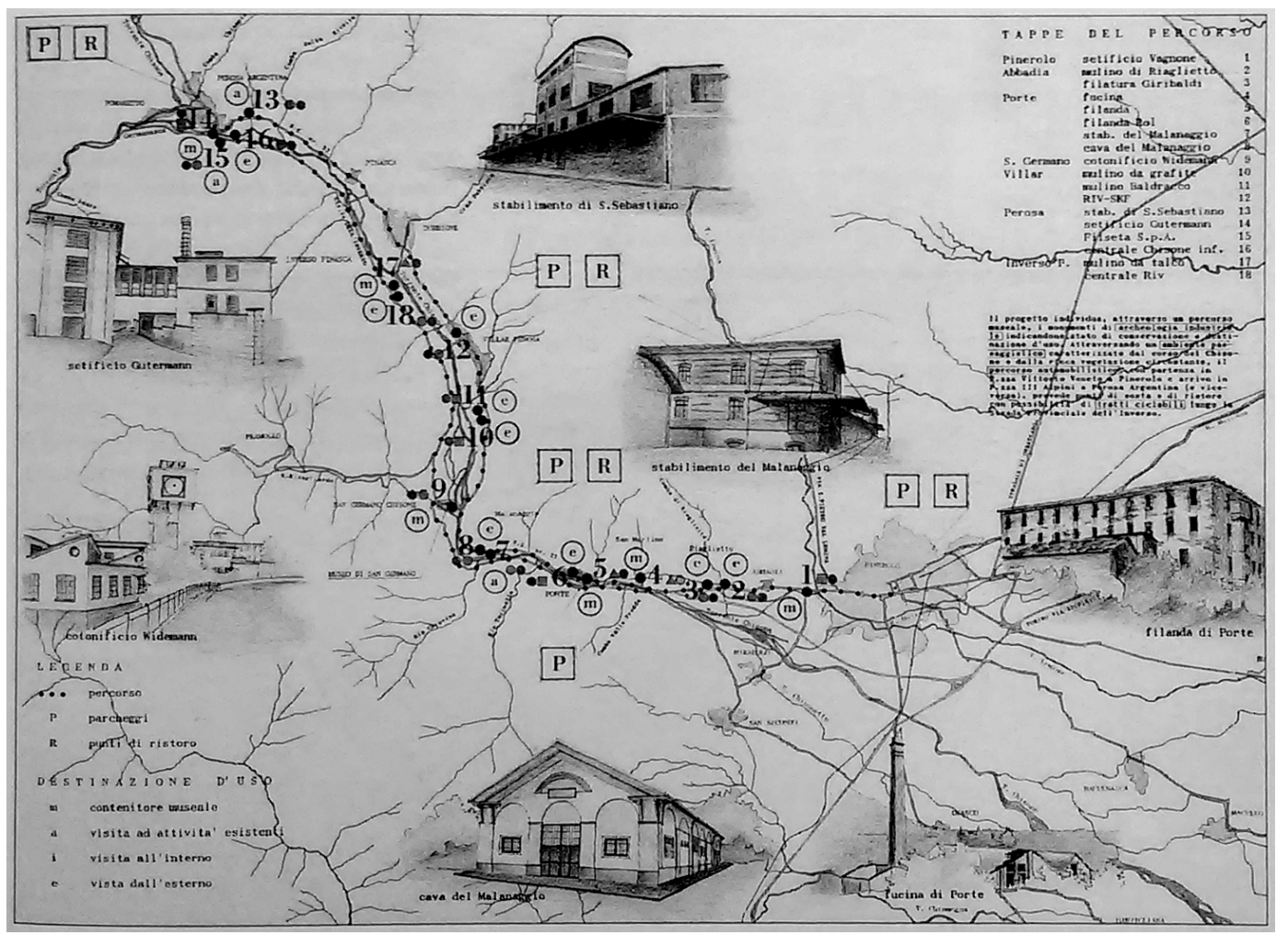

Figura 16. Progetti di sistematizzazione del patrimonio industriale pinerolese e valligiano: il percorso museale Pinerolo-Perosa Argentina proposto nel libro I luoghi del lavoro nel Pinerolese (Cerrato \& Ronchetta, 1996, p. 187). 
Il progetto s'inserisce in un contesto che vede la costituzione di un polo di media-bassa Val Chisone, legato in particolare alle Famiglie Widemann, Gutermann e Agnelli (che, per la sua indubbia fama, costituisce un bene faro con grande capacità attrattiva). Questo sistema andrebbe poi messo in relazione con gli altri presenti sul territorio, comprendendo la Val Pellice, Pinerolo (con il suo sistema di industrie e proto-industrie sul rio Moirano, canale artificiale di origine medievale motore delle attività industriali cittadine) e il Pinerolese, ed estendendosi fino all'Alta Val Susa, la Val Po e la Francia.

Il progetto, peraltro, può considerarsi il volano per la promozione territoriale su più ampia scala: infatti, il percorso industriale lungo il Chisone e i suoi principali affluenti può essere il polo attrattore per altri itinerari, da quello naturalistico a quello legato ai siti pre e protostorici, senza trascurare la possibile valorizzazione dei borghi storici e degli antichi nuclei abitativi delle Valli. Si potranno inoltre promuovere i percorsi di visita già esistenti, collegandoli organicamente con altri nuovi, incrementando la "mobilità dolce" e lo sviluppo di itinerari alternativi a quelli viari ad alto scorrimento. Poiché la val Chisone si trova lungo uno degli assi di accesso all'Italia dalla Francia consente di coinvolgere anche i visitatori transalpini che già naturalmente transitano in valle. Inoltre la vicinanza alle grandi stazioni sciistiche (Sestriere) e a Torino, consentono di disporre di un bacino di visitatori decisamente consistente. Peraltro, in Val Chisone verrà a breve resa praticabile la "Route d'Artagnan"33, progetto che coinvolge sei Stati europei che ha come capofila la Federazione Equituristica Francese e che aprirà Pinerolo a un turismo internazionale.

Questo contesto multidisciplinare, quindi, dove la cultura, la partecipazione diretta della popolazione in generale e delle scuole in particolare e l'attività turistica sono punti focali, può costituire una valida occasione per generare nuove prospettive di sviluppo socio-economico della Valle, legate indissolubilmente a una più consapevole conoscenza del territorio e delle sue molteplici risorse.

\section{Referenze}

Avondo, G. V., Bruno, V., \& Seglie, D. (1998). C'era una volta...il Gibuti. Pinerolo: Alzani.

Balcet, G. (2014). Imprenditori esteri e imprese multinazionali nella storia industriale delle Valli negli ultimi due secoli. In C. Bermond (a cura di). Una montagna viva. Mondo rurale, industria e turismo nelle Valli pinerolesi nei secoli XVII-XX. Perosa Argentina: LAR editore, pp. 115-127.

Bermond, C. (2014). L'evoluzione economica e sociale delle Valli dal Seicento al Novecento. In C. Bermond (a cura di). Una montagna viva. Mondo rurale, industria e turismo nelle Valli pinerolesi nei secoli XVII-XX. Perosa Argentina: LAR editore, p. 57-111.

Bottazzi, A., \& Bounous C. (1994). Quando la sirena suonava... il cotonificio di S. Germano Chisone in un secolo di Storia. Pinerolo: Alzani.

Bourlot, R. (2014). La Società Anonima Cooperativa Fenestrellese per l'illuminazione elettrica (1893-1960). In C. Bermond (a cura di). Una montagna viva. Mondo rurale, industria e turismo nelle Valli pinerolesi nei secoli XVII-XX. Perosa Argentina: LAR editore, pp. 219-235.

Campra, F. (2000). La funzionalità del torrente Chisone in una prospettiva di sfruttamento idroelettrico. Tesi di Laurea in Scienze matematiche, fisiche e naturali, Università degli Studi di Torino, Rel. Prof. Roberto Ajassa, A.A. $1999-2000$.

Cerrato A., \& Ronchetta C. (a cura di) (1996). I luoghi del lavoro nel Pinerolese. Tra mulini e fabbriche, centrali e miniere. Torino: Celid.

Massarente, A., \& Ronchetta, C. (a cura di) (2004). Ecomusei e paesaggi. Esperienze, progetti e ricerche per la cultura materiale. Milano: Lybra immagine.

\footnotetext{
33 https://www.route-dartagnan.eu/
} 
Romeo, E. (a cura di) (2015). Memoria, conservazione, riuso del patrimonio industriale. Il caso studio dell'IPCA di Cirié. Roma: Ermes edizioni scientifiche.

Ronchetta, C., \& Trisciuoglio, M. (a cura di) (2008). Progettare per il patrimonio industriale. Torino: Celid.

Sibilla, P. (2014). Tenuta e declino del mondo rurale alpino nei secoli XIX e XX. In C. Bermond (a cura di). Una montagna viva. Mondo rurale, industria e turismo nelle Valli pinerolesi nei secoli XVII-XX. Perosa Argentina: LAR editore, p. 27-54.

Tourn, G. (2003). I valdesi: identità e storia. Torino: Claudiana. 